\title{
On positive solutions to equations involving the one-dimensional $p$-Laplacian
}

\author{
Ruyun Ma*, Yanqiong Lu and Ahmed Omer Mohammed Abubaker
}

"Correspondence:

mary@nwnu.edu.cn

Department of Mathematics,

Northwest Normal University,

Lanzhou, 730070, P.R. China

\begin{abstract}
We consider equations involving the one-dimensional $p$-Laplacian$$
\left(\left|u^{\prime}(t)\right|^{p-2} u^{\prime}(t)\right)^{\prime}+\lambda f(u(t))=0, \quad t \in(0,1)
$$

with the Dirichlet boundary conditions. By using time map methods, we show how changes of the sign of $f(\cdot)$ lead to multiple positive solutions of the problem for sufficiently large $\lambda$.
\end{abstract}

MSC: 34B10; 34B18

Keywords: positive solutions; one-dimensional p-Laplacian; uniqueness; time map

\section{Introduction}

Let $f:[0, \infty) \rightarrow \mathbb{R}$ be continuous and change its sign. Let $\Omega$ be an open subset of $\mathbb{R}^{N}$ with smooth boundary $\partial \Omega$. The semi-positone problems and their special cases

$$
\Delta u+\lambda f(u)=0 \quad \text { in } \Omega, \quad u=0 \quad \text { on } \partial \Omega
$$

and

$$
u^{\prime \prime}(t)+\lambda f(u(t))=0, \quad t \in(0,1), \quad u(0)=u(1)=0
$$

(and their finite difference analogues) have been extensively studied since early 1980s. Several different approaches such as variational methods, bifurcation theory, lower and upper solutions method and quadrature arguments have been successfully applied to show the existence of multiple solutions. See Brown and Budin [1], Peitgen et al. [2], Peitgen and Schmitt [3], Hess [4], Ambrosetti and Hess [5], Cosner and Schmitt [6], Dancer and Schmitt [7], Espinoza [8], Anuradha and Shivaji [9], Anuradha et al. [10], de Figueiredo [11], Lin and Pai [12], Clément and Sweers [13] and the references therein.

Very recently, Loc and Schmitt [14] considered the problem

$$
\Delta_{p} u+\lambda f(u)=0 \quad \text { in } \Omega, \quad u=0 \quad \text { on } \partial \Omega,
$$

where $\Delta_{p}$ is the $p$-Laplace operator for $p \in(1, \infty)$. They assumed that the nonlinearity $f$ is a continuous function on $\mathbb{R}, f(0) \geq 0$, and there exist $0<a_{1}<b_{1}<a_{2}<b_{2}<\cdots<b_{m-1}<a_{m}$

\section{Springer}

(c) 2013 Ma et al.; licensee Springer. This is an Open Access article distributed under the terms of the Creative Commons Attribution License (http://creativecommons.org/licenses/by/2.0), which permits unrestricted use, distribution, and reproduction in any medium, provided the original work is properly cited. 
such that $f \leq 0$ on $\left(a_{k}, b_{k}\right)$ and $f \geq 0$ on $\left(b_{k}, a_{k+1}\right)$ for every $k=1, \ldots, m-1$. They proved that, for $\lambda$ sufficiently large, if

$$
\int_{a_{k}}^{a_{k+1}} f(s) d s>0 \quad \text { for all } k \in\{1, \ldots, m-1\},
$$

then the problem (1.3) has at least $m-1$ positive bounded solutions $u_{1}, \ldots, u_{m-1}$ which belong to the Sobolev space $W_{0}^{1, p}(\Omega)$ and are such that $\|u\|_{\infty} \in\left(a_{k}, a_{k+1}\right]$ for each $k \in$ $\{1, \ldots, m-1\}$, where

$$
\|u\|_{\infty}=\max \{|u(x)| \mid x \in \bar{\Omega}\} .
$$

In the special case that $p=2$ and $N=1$, Brown and Budin [1] applied the quadrature arguments to get the following more detailed results.

Theorem A [1, Theorem 3.8] Assume that

(H1) $f \in C^{1}[0, \infty)$;

(H2) $f(0)>0$;

(H3) There exists $a_{1}, \ldots, a_{n} \in \mathbb{R}$ such that $0<a_{1}<a_{2}<\cdots<a_{n}$ and $f\left(a_{i}\right) \leq 0$ for $i=1,2, \ldots, n$;

(H4) If $F(u)=\int_{0}^{u} f(s) d s$, there exist $b_{1}, \ldots, b_{n-1} \in \mathbb{R}$ with $a_{1}<b_{1}<a_{2}<b_{2}<\cdots<a_{n-1}<b_{n-1}<a_{n}$ such that $f\left(b_{i}\right)>0$ and $F\left(b_{i}\right)>F(u)$ for $0 \leq u \leq b_{i}, i=1,2, \ldots, n-1$.

Then:

(a) For all $\lambda>0$, there exists a solution $(\lambda, u)$ of (1.2).

(b) If $\lambda>\inf \left\{\lambda(\rho): \rho \in\left(\alpha_{i}, \beta_{i}\right)\right\}$, there exist at least two solutions $(\lambda, u)$ of $(1.2)$ such that

$$
\alpha_{i}<\|u\|_{\infty}<\beta_{i}, \quad i=1,2, \ldots, n-1,
$$

where

$$
\beta_{i}=\inf \left\{u>b_{i} \mid f(u)=0\right\}, \quad \alpha_{i}=\inf \left\{u \mid\left(u, \beta_{i}\right) \subseteq S\right\}
$$

and

$$
\mathcal{S}=\{u \mid u>0, f(u)>0, F(u)>F(s) \text { for all } s: 0 \leq s<u\} .
$$

(c) If $(\lambda, u)$ is any solution of $(1.2)$ such that $\alpha_{i}<\|u\|_{\infty}<\beta_{i}$, then

$$
\lambda>4 \alpha_{i} k^{-1},
$$

where $k=\sup \left\{|f(u)|: 0 \leq u \leq \beta_{i}\right\}$

Of course the natural question is whether or not the similar results still hold for the corresponding problem involving the one-dimensional $p$-Laplacian

$$
\begin{aligned}
& \left(\left|u^{\prime}(t)\right|^{p-2} u^{\prime}(t)\right)^{\prime}+\lambda f(u(t))=0, \quad t \in(0,1), \\
& u(0)=u(1)=0 .
\end{aligned}
$$


We shall answer these questions in the affirmative if $p \in(1,2]$. More precisely, we get the following theorem.

Theorem 1.1 Let $p \in(1,2]$ and let (H1), (H3), (H4) hold. Assume that

$\left(\mathrm{H} 2^{\prime}\right)$ either $f(0)>0$ or $f(0)=0$ and

$$
f_{0}=\lim _{s \rightarrow 0^{+}} \frac{f(s)}{s^{p-1}}>0 .
$$

Then:

(a) For all $\lambda>\frac{\lambda_{1}}{f_{0}}$, there exists a solution $(\lambda, u)$ of $(1.7)$, and $\lambda_{1}$ is the least eigenvalue of $B V P$

$$
\begin{aligned}
& \left(\left|u^{\prime}(t)\right|^{p-2} u^{\prime}(t)\right)^{\prime}+\lambda|u(t)|^{p-2} u(t)=0, \quad t \in(0,1), \\
& u(0)=u(1)=0 .
\end{aligned}
$$

(b) If $\lambda>\inf \left\{\lambda(\rho): \rho \in\left(\alpha_{i}, \beta_{i}\right)\right\}$, there exist at least two solutions $(\lambda, u)$ of $(1.7)$ such that

$$
\alpha_{i}<\|u\|_{\infty}<\beta_{i}, \quad i=1,2, \ldots, n-1 .
$$

(c) If $(\lambda, u)$ is any solution of (1.7) such that $\alpha_{i}<\|u\|_{\infty}<\beta_{i}$, then

$$
\lambda>\left(\frac{\alpha_{i}}{C_{*}}\right)^{p-1},
$$

where

$$
C_{*}=\frac{p-1}{p} \cdot\left(\frac{1}{2}\right)^{\frac{p}{p-1}}\left(\sup _{s \in\left[0, \beta_{i}\right]}|f(s)|\right)^{\frac{1}{p-1}}
$$

We shall apply the time map method to show how changes of the sign of $f(\cdot)$ lead to multiple positive solutions of (1.7) for sufficiently large $\lambda$.

In the following, we extend $f$ so that $f(u)>0$ for all $u<0$, then all the solutions of (1.7) are positive on $(0,1)$.

\section{Preliminaries}

To prove our main results, we use the uniqueness results due to Reichel and Walter [15] on the initial value problem

$$
\begin{aligned}
& \left(\left|u^{\prime}(t)\right|^{p-2} u^{\prime}(t)\right)^{\prime}+\lambda f(u(t))=0, \quad t \in(0,1), \\
& u(a)=b, \quad u^{\prime}(a)=d
\end{aligned}
$$

where $a \in[0,1]$ and $b, d \in \mathbb{R}$.

Lemma 2.1 Let $(\mathrm{H} 1)$ hold. If $a \in(0,1]$ and $d \neq 0$, then the initial value problem (2.1) has a unique local solution. The extension $u(t)$ remains unique as long as $u^{\prime}(t) \neq 0$.

Proof It is an immediate consequence of Reichel and Walter [15, Theorem 2]. 
Lemma 2.2 Let $(\mathrm{H} 1)$ hold. Let $a \in(0,1)$, and let $\rho \in(0, \infty)$ be such that

$$
f(\rho) \neq 0 \text {. }
$$

Then the initial value problem

$$
\begin{aligned}
& \left(\left|u^{\prime}(t)\right|^{p-2} u^{\prime}(t)\right)^{\prime}+\lambda f(u(t))=0, \quad t \in(0,1), \\
& u(a)=\rho, \quad u^{\prime}(a)=0
\end{aligned}
$$

has a unique local solution.

Proof (H1) implies that $f$ is locally Lipschitzian. This together with the assumption $f(\rho) \neq$ 0 and using [15, (iii) and (v) in the case $(\beta)$ of Theorem 4] yields that (2.2) has a unique solution in some neighborhood of $a$.

Lemma 2.3 Let $g: \mathbb{R} \rightarrow \mathbb{R}$ be continuous. Let $u$ be a solution of the equation

$$
\left(\left|u^{\prime}(t)\right|^{p-2} u^{\prime}(t)\right)^{\prime}+g(u(t))=0, \quad t \in(0,1)
$$

with $\|u\|_{\infty}=\rho \in \mathcal{S}$. Let $x_{0} \in(0,1)$ be such that $u^{\prime}\left(x_{0}\right)=0$. Then

$$
u\left(x_{0}-t\right) \equiv u\left(x_{0}+t\right), \quad t \in\left(0, \min \left\{x_{0}, 1-x_{0}\right\}\right)
$$

Proof Since $g$ is independent of $t$, both $u\left(x_{0}-t\right)$ and $u\left(x_{0}+t\right)$ satisfy the initial value problem

$$
\left\{\begin{array}{l}
\left(\left|w^{\prime}(t)\right|^{p-2} w^{\prime}(t)\right)^{\prime}+g(w(t))=0, \quad t \in\left(0, \min \left\{x_{0}, 1-x_{0}\right\}\right) \\
w(0)=u\left(x_{0}\right) \\
w^{\prime}(0)=0
\end{array}\right.
$$

By Lemmas 2.1 and $2.2,(2.5)$ has a unique solution defined on $t \in\left(0, \min \left\{x_{0}, 1-x_{0}\right\}\right)$. Therefore, (2.4) is true.

Lemma 2.4 Let $(\lambda, u)$ be a positive solution of the problem

$$
\begin{aligned}
& \left(\left|u^{\prime}(t)\right|^{p-2} u^{\prime}(t)\right)^{\prime}+\lambda f(u(t))=0, \quad t \in(0,1), \\
& u(0)=u(1)=0
\end{aligned}
$$

with $\|u\|_{\infty}=\rho \in \mathcal{S}$ and $\lambda>0$. Let $x_{0} \in(0,1)$ be such that $u^{\prime}\left(x_{0}\right)=0$. Then

(a) $x_{0}=\frac{1}{2}$

(b) $x_{0}$ is the unique point on which $u$ attains its maximum;

(c) $u^{\prime}(t)>0, t \in\left(0, \frac{1}{2}\right)$.

Proof (a) Suppose on the contrary that $x_{0} \neq \frac{1}{2}$, say $x_{0}>\frac{1}{2}$, then

$$
0=u(1)=u\left(1-2 x_{0}\right)
$$

However, this is impossible since $1-2 x_{0} \in(0,1)$ and $u>0$ in $(0,1)$. Therefore $x_{0}=\frac{1}{2}$. 
(b) Suppose on the contrary that there exists $x_{1} \in(0,1)$ with $x_{1} \neq x_{0}$ and

$$
u\left(x_{1}\right)=u\left(x_{0}\right)=: \rho .
$$

We may assume that $x_{1}<x_{0}$. The other case can be treated in a similar way.

If $u(t) \equiv u\left(x_{0}\right)$ in the interval $\left(x_{1}, x_{0}\right)$, then Lemma 2.3 yields that

$$
u(t) \equiv u\left(x_{0}\right)=\rho>0, \quad t \in(0,1) .
$$

This contradicts the boundary conditions $u(0)=u(1)=0$. Therefore, $u(t) \not \equiv u\left(x_{0}\right)$ in any subinterval of $(0,1)$.

So, there exists $x_{*} \in\left(x_{1}, x_{0}\right)$, such that

$$
u\left(x_{*}\right)=\min \left\{u(t) \mid t \in\left(x_{1}, x_{0}\right)\right\}
$$

Obviously,

$$
0<u\left(x_{*}\right)<\rho, \quad u^{\prime}\left(x_{*}\right)=0 .
$$

Multiplying both sides of the equation in (2.6) by $u^{\prime}$ and integrating from $t$ to $x_{0}$, we get that

$$
\left|u^{\prime}(t)\right|^{p}=\lambda \frac{p}{p-1}[F(\rho)-F(u(t))], \quad t \in\left[0, \frac{1}{2}\right]
$$

and subsequently,

$$
0=\left|u^{\prime}\left(x_{*}\right)\right|^{p}=\lambda \frac{p}{p-1}\left[F(\rho)-F\left(u\left(x_{*}\right)\right)\right]
$$

This contradicts the facts that $\rho \in \mathcal{S}$ and $u\left(x_{*}\right)<\rho$. Therefore,

$$
u\left(\frac{1}{2}\right)>u(t), \quad t \in\left[0, \frac{1}{2}\right)
$$

Similarly, we can prove that

$$
u\left(\frac{1}{2}\right)>u(t), \quad t \in\left(\frac{1}{2}, 1\right]
$$

(c) Suppose on the contrary that there exists $\hat{x} \in\left(0, \frac{1}{2}\right)$ with $u^{\prime}(\hat{x})=0$. Then

$$
u(\hat{x})<\rho .
$$

This together with (2.8) implies that

$$
0=\left|u^{\prime}(\hat{x})\right|^{p}=\lambda \frac{p}{p-1}[F(\rho)-F(u(\hat{x}))]
$$

This contradicts the facts that $\rho \in \mathcal{S}$ and $u(\hat{x})<\rho$. 


\section{Proof of the main results}

To prove Theorem 1.1, we need the following preliminary results.

Lemma 3.1 For any $\rho \in \mathcal{S}$, there exists a unique $\lambda>0$ such that

$$
\begin{aligned}
& \left(\left|u^{\prime}(t)\right|^{p-2} u^{\prime}(t)\right)^{\prime}+\lambda f(u(t))=0, \quad t \in(0,1), \\
& u(0)=u(1)=0
\end{aligned}
$$

has a positive solution $(\lambda, u)$ with $\|u\|_{\infty}=\rho$. Moreover, $\rho \rightarrow \lambda(\rho)$ is a continuous function on $\mathcal{S}$.

Proof By Lemma 2.4, $(\lambda, u)$ is a positive solution of (3.1), (3.2) if and only if $(\lambda, u)$ is a positive solution of

$$
\begin{aligned}
& \left(\left|u^{\prime}(t)\right|^{p-2} u^{\prime}(t)\right)^{\prime}+\lambda f(u(t))=0, \quad t \in\left(0, \frac{1}{2}\right), \\
& u(0)=u^{\prime}\left(\frac{1}{2}\right)=0 .
\end{aligned}
$$

Suppose that $(\lambda, u)$ is a solution of (3.3), (3.4) with $\|u\|_{\infty}=\rho$. Then

$$
\left|u^{\prime}(t)\right|^{p}=\lambda \frac{p}{p-1}(F(\rho)-F(u(t))), \quad t \in\left[0, \frac{1}{2}\right]
$$

and so

$$
t\left(\frac{p}{p-1} \lambda\right)^{1 / p}=\int_{0}^{u(t)}(F(\rho)-F(s))^{-1 / p} d s, \quad t \in\left[0, \frac{1}{2}\right] .
$$

Putting $t=\frac{1}{2}$, we obtain

$$
\lambda^{1 / p}=2\left(\frac{p-1}{p}\right)^{1 / p} \int_{0}^{\rho}(F(\rho)-F(s))^{-1 / p} d s .
$$

Hence $\lambda$ (if exists) is uniquely determined by $\rho$.

If $\rho \in \mathcal{S}$, we define $\lambda(\rho)$ by (3.6) and $u(t)$ by (3.5). It is straightforward to verify that $u$ is twice differentiable, $u$ satisfies (3.3), (3.4), $u>0$ in $(0,1)$ and $u(1 / 2)=\rho$. The continuity of $\lambda(\cdot)$ is implied by (3.6) and this completes the proof.

Let

$$
r=\inf \{u>0: f(u)=0\}
$$

Then $(0, r) \in \mathcal{S}$.

Lemma 3.2 Let $(\mathrm{H} 1)$ and $\left(\mathrm{H} 2^{\prime}\right)$ hold, and let $p \in(1, \infty)$. Then

$$
\lim _{\rho \rightarrow 0} \lambda(\rho)=\frac{\lambda_{1}}{f_{0}}, \quad \lim _{\rho \rightarrow r^{-}} \lambda(\rho)=\infty
$$

where $\lambda_{1}$ is the least eigenvalue of (1.9). 
Proof We only deal with $\lim _{\rho \rightarrow 0} \lambda(\rho)=\frac{\lambda_{1}}{f_{0}}$. The other one can be treated by the same method.

To this end, we divide the proof into two cases.

Case 1. We show that $f_{0}=\infty$ implies $\lim _{\rho \rightarrow 0} \lambda(\rho)=0$.

In this case, for any $M>0$, there is a positive number $R$ such that $f(w)>M w^{p-1}$ for $0 \leq w \leq R$. Thus, if $\rho<R$, then

$$
\begin{aligned}
F(\rho)-F(w) & =\int_{w}^{\rho} f(v) d v \\
& \geq \frac{M}{p}\left(\rho^{p}-w^{p}\right)
\end{aligned}
$$

for $0 \leq w \leq \rho$. From (3.6), we have that for any $\rho \leq R$,

$$
\begin{aligned}
{[\lambda(\rho)]^{\frac{1}{p}} } & =2\left(\frac{p-1}{p}\right)^{\frac{1}{p}} \int_{0}^{\rho} \frac{d w}{[F(\rho)-F(w)]^{1 / p}} \\
& \leq 2\left(\frac{p-1}{p}\right)^{1 / p}\left(\frac{p}{M}\right)^{1 / p} \int_{0}^{\rho} \frac{d w}{\left[\rho^{p}-w^{p}\right]^{1 / p}} \\
& \leq 2\left(\frac{p-1}{M}\right)^{1 / p} \int_{0}^{1} \frac{d \frac{w}{\rho}}{\left[1-\left(\frac{w}{\rho}\right) p^{p}\right]^{1 / p}} \\
& \leq 2\left(\frac{p-1}{M}\right)^{1 / p}\left(\frac{1}{p-1}\right)^{1 / p} \int_{0}^{(p-1)^{1 / p}} \frac{d s}{\left[1-\frac{s^{p}}{p-1}\right]^{1 / p}} \\
& \leq 2\left(\frac{1}{M}\right)^{1 / p} \int_{0}^{(p-1)^{1 / p}} \frac{d s}{\left[1-\frac{s^{p}}{p-1}\right]^{1 / p}} \\
& =\left(\frac{1}{M}\right)^{1 / p} \pi_{p},
\end{aligned}
$$

where

$$
\pi_{p}:=2 \int_{0}^{(p-1)^{1 / p}} \frac{d s}{\left[1-\frac{s^{p}}{p-1}\right]^{1 / p}},
$$

see Zhang [16]. Hence

$$
\lim _{\rho \rightarrow 0} \lambda(\rho)=0
$$

Case 2. We show that $f_{0}=m$ for some $m \in(0, \infty)$ implies that

$$
\lim _{\rho \rightarrow 0} \lambda(\rho)=\frac{p-1}{p m} \tau_{p}^{p}=\frac{\left(\pi_{p}\right)^{p}}{f_{0}},
$$

where

$$
\tau_{p}=2 \int_{0}^{1}\left[\frac{p}{1-v^{p}}\right]^{1 / p} d v, \quad p>1
$$


In fact, (3.6) yields

$$
\begin{aligned}
{[m \lambda(\rho)]^{1 / p}=} & 2\left[\frac{m(p-1)}{p}\right]^{\frac{1}{p}} \int_{0}^{\rho} \frac{d w}{[F(\rho)-F(w)]^{1 / p}} \\
= & 2\left(\frac{p-1}{p}\right)^{1 / p} \cdot \int_{0}^{1}\left[\frac{p}{1-v^{p}}\right]^{1 / p} d v \\
& -2\left(\frac{p-1}{p}\right)^{1 / p} \int_{0}^{1}\left[\frac{p}{1-v^{p}}\right]^{1 / p} \frac{[1+\gamma(\rho, v)]^{1 / p}-1}{[1+\gamma(\rho, v)]^{1 / p}} d v
\end{aligned}
$$

for $p>1$, where

$$
\gamma(\rho, v)=\frac{p}{m} \frac{\int_{\rho \nu}^{\rho}\left[f(w)-m w^{p-1}\right] d w}{\rho^{p}\left(1-v^{p}\right)} .
$$

We will show that the last integral in (3.9) converges to zeros as $\rho \rightarrow 0$.

For $0 \leq v \leq \frac{1}{2}$, using l'Hospital's rule, it follows that as $\rho \rightarrow 0$,

$$
\begin{aligned}
|\gamma(\rho, v)| & =\frac{p}{m} \frac{\int_{\rho v}^{\rho}\left|f(w)-m w^{p-1}\right| d w}{\rho^{p}\left(1-v^{p}\right)} \\
& \leq \frac{p}{m} \frac{\int_{0}^{\rho}\left|f(w)-m w^{p-1}\right| d w}{\rho^{p}\left(1-v^{p}\right)} \\
& \rightarrow \frac{p}{m} \frac{\left|f(\rho)-m \rho^{p-1}\right|}{p \rho^{p-1}\left(1-v^{p}\right)} \\
& =\frac{p}{m} \frac{\left|\frac{f(\rho)}{\rho^{p-1}}-m\right|}{p\left(1-v^{p}\right)} \\
& \rightarrow 0 .
\end{aligned}
$$

For $\frac{1}{2} \leq v \leq 1$,

$$
\lim _{\rho \rightarrow 0}|\gamma(\rho, v)|=\limsup _{\rho \rightarrow 0}\left\{\left|\frac{f(w)-m w^{p-1}}{w^{p-1}}\right|: \frac{1}{2} \rho \leq w \leq \rho\right\} \frac{p}{m \rho^{p}\left(1-v^{p}\right)} \int_{\rho v}^{\rho} w^{p-1} d w=0
$$

uniformly in $v$. Therefore, (3.9) implies

$$
\lim _{\rho \rightarrow 0}[m \lambda(\rho)]^{1 / p}=2\left(\frac{p-1}{p}\right)^{\frac{1}{p}} \int_{0}^{1}\left[\frac{p}{1-v^{p}}\right]^{1 / p} d \nu
$$

Therefore, (3.7) holds.

From the definitions of $\alpha_{i}$ and $\beta_{i}$, we have that $a_{i} \leq \alpha_{i}<\beta_{i} \leq a_{i+1}$ and $\left(\alpha_{i}, \beta_{i}\right) \subseteq \mathcal{S}$ for $i=1,2, \ldots, n-1$. Moreover, we have the following.

Lemma 3.3 Let $p \in(1,2]$. Then

(i) $\lim _{\rho \rightarrow \alpha_{i}^{+}} \lambda(\rho)=\infty$;

(ii) $\lim _{\rho \rightarrow \beta_{i}^{-}} \lambda(\rho)=\infty$. 
Proof (i) Suppose firstly that $f\left(\alpha_{i}\right)>0$. Since $S$ is open, $\alpha_{i} \notin S$ and so there exists $k$ : $0<k<$ $\alpha_{i}$ such that

$$
F\left(\alpha_{i}\right)=F(k) .
$$

Clearly $k$ must be a local maximum for $F$ and so $f(k)=0$. If $M=\max \left\{\left|f^{\prime}(u)\right|: 0 \leq u \leq b_{i}\right\}$, then

$$
f(u) \leq M|u-k|, \quad 0 \leq u \leq b_{i}
$$

Let

$$
N=\max \left\{|f(u)|: 0 \leq u \leq b_{i}\right\} .
$$

Then if $\alpha_{i}<\rho<b_{i}$,

$$
\begin{aligned}
F(\rho)-F(u)= & F(\rho)-F\left(\alpha_{i}\right)+F(k)-F(u) \\
= & \left(\rho-\alpha_{i}\right) f(\xi)+(k-u) f(\eta), \\
& \text { where } \xi \in\left(\alpha_{i}, \rho\right) \text { and } \eta \in(k, u) \\
\leq & N\left(\rho-\alpha_{i}\right)+M(k-u)^{2} .
\end{aligned}
$$

Hence

$$
\begin{aligned}
(\lambda(\rho))^{1 / p} & =2\left(\frac{p-1}{p}\right)^{1 / p} \int_{0}^{\rho}(F(\rho)-F(s))^{-1 / p} d s \\
& \geq 2\left(\frac{p-1}{p}\right)^{1 / p} \int_{0}^{\alpha_{i}}\left(N\left(\rho-\alpha_{i}\right)+M(k-u)^{2}\right)^{-1 / p} d u \\
& =\int_{0}^{\alpha_{i}} H_{\rho}(u) d u .
\end{aligned}
$$

As $\rho \rightarrow \alpha_{i}^{+}, H_{\rho}(u)=2\left(\frac{p-1}{p}\right)^{1 / p}\left(N\left(\rho-\alpha_{i}\right)+M(k-u)^{2}\right)^{-1 / p}$ is a nondecreasing sequence of measurable functions. Therefore, by the monotone convergence theorem and the assumption $p \geq 2$, it follows that

$$
\begin{aligned}
\lim _{\rho \rightarrow \alpha_{i}^{+}}[\lambda(\rho)]^{1 / p} & \geq \lim _{\rho \rightarrow \alpha_{i}^{+}} \int_{0}^{\alpha_{i}} H_{\rho}(u) d u \\
& =\int_{0}^{\alpha_{i}} 2\left(\frac{p-1}{p}\right)^{1 / p} M^{-1 / p}[k-u]^{-2 / p} d u=\infty
\end{aligned}
$$

since $k \in\left(0, \alpha_{i}\right)$.

Suppose next that $f\left(\alpha_{i}\right)=0$. Then $F^{\prime}\left(\alpha_{i}\right)=0$.

Since

$$
\begin{aligned}
& F\left(\alpha_{i}\right)-F(u)=f(\eta)\left(\alpha_{i}-u\right), \quad \text { where } \eta \in\left(u, \alpha_{i}\right), \\
& |f(u)|=\left|f(u)-f\left(\alpha_{i}\right)\right| \leq M\left|u-\alpha_{i}\right| .
\end{aligned}
$$


Thus

$$
\begin{aligned}
\int_{0}^{\alpha_{i}}\left[F\left(\alpha_{i}\right)-F(u)\right]^{-1 / p} d u & \geq \int_{0}^{\alpha_{i}}\left[M\left|\alpha_{i}-u\right|^{2}\right]^{-1 / p} d u \\
& =\int_{0}^{\alpha_{i}} M^{-1 / p}\left|\alpha_{i}-u\right|^{-2 / p} d u=\infty
\end{aligned}
$$

(ii) Let $K_{1}=\max \left\{|f(u)|: 0 \leq u \leq \beta_{i}\right\}$ and $K_{2}=\max \left\{\left|f^{\prime}(u)\right|: 0 \leq u \leq \beta_{i}\right\}$. Since $f\left(\beta_{i}\right)=0$,

$$
f(u) \leq K_{2}\left|u-\beta_{i}\right|, \quad 0 \leq u<\beta_{i} .
$$

Hence, if $0 \leq u \leq \rho<\beta_{i}$, then it follows from (3.11) that

$$
\begin{aligned}
F(\rho)-F(u)= & F(\rho)-F\left(\beta_{i}\right)+F\left(\beta_{i}\right)-F(u) \\
= & \left(\rho-\beta_{i}\right) f(\xi)+\left(\beta_{i}-u\right) f(\eta), \\
& \text { where } \xi \in\left(\rho, \beta_{i}\right), \eta \in\left(u, \beta_{i}\right) \\
\leq & K_{1}\left(\beta_{i}-\rho\right)+K_{2}\left(\beta_{i}-u\right)^{2} .
\end{aligned}
$$

Hence, if $0<\rho<\beta_{i}$,

$$
\begin{aligned}
(\lambda(\rho))^{1 / p} & \geq 2\left(\frac{p-1}{p}\right)^{1 / p} \int_{0}^{\rho}\left(K_{1}\left(\beta_{i}-\rho\right)+K_{2}\left(\beta_{i}-u\right)^{2}\right)^{-1 / p} d u \\
& =\int_{0}^{\beta_{i}} G_{\rho}(u) d u,
\end{aligned}
$$

where $G_{\rho}(u)=2\left(\frac{p-1}{p}\right)^{1 / p}\left(K_{1}\left(\beta_{i}-\rho\right)+K_{2}\left(\beta_{i}-u\right)^{2}\right)^{-1 / p} \chi_{[0, \rho]}$ and $\chi_{[0, \rho]}$ denotes the characteristic function of $[0, \rho]$. As $G_{\rho}$ is a nondecreasing sequence of measurable functions, by the monotone convergence theorem

$$
\begin{aligned}
\lim _{\rho \rightarrow \beta_{i}^{-}}[\lambda(\rho)]^{1 / p} & \geq \lim _{\rho \rightarrow \beta_{i}^{-}} \int_{0}^{\beta_{i}} G_{\rho}(u) d u \\
& =\int_{0}^{\beta_{i}} 2\left(\frac{p-1}{p}\right)^{1 / p} K_{2}^{-1 / p}\left|\beta_{i}-u\right|^{-2 / p} d u=\infty
\end{aligned}
$$

Proof of Theorem 1.1 (a) follows from the continuity of $\rho \rightarrow \lambda(\rho)$ and Lemma 3.2.

(b) follows from the continuity of $\rho \rightarrow \lambda(\rho)$ and Lemma 3.3.

(c) $(\lambda, u)$ is any solution of (3.1), (3.2) if and only if

$$
u(t)=\int_{0}^{t}\left(\int_{\tau}^{1 / 2} \lambda f(u(s)) d s\right)^{\frac{1}{p-1}} d \tau, \quad t \in\left[0, \frac{1}{2}\right]
$$

Hence

$$
\begin{aligned}
|u(t)| & \leq \lambda^{\frac{1}{p-1}} \int_{0}^{1 / 2}\left(\int_{\tau}^{1 / 2}|f(u(s))| d s\right)^{\frac{1}{p-1}} d \tau \\
& \leq \lambda^{\frac{1}{p-1}} \frac{p-1}{p} \cdot\left(\frac{1}{2}\right)^{\frac{p}{p-1}}\left(\sup _{y \in[0,1]}|f(u(y))|\right)^{\frac{1}{p-1}} .
\end{aligned}
$$


Now, if $\alpha_{i}<\|u\|_{\infty}<\beta_{i}$, then

$$
\alpha_{i} \leq \lambda^{\frac{1}{p-1}} \frac{p-1}{p} \cdot\left(\frac{1}{2}\right)^{\frac{p}{p-1}}\left(\sup _{s \in\left[0, \beta_{i}\right]}|f(s)|\right)^{\frac{1}{p-1}}
$$

and so

$$
\lambda>\left(\frac{\alpha_{i}}{C_{*}}\right)^{p-1} .
$$

\section{Competing interests}

The authors declare that they have no competing interests.

\section{Authors' contributions}

RM completed the main study, carried out the results of this article. YL drafted the manuscript. AOMA checked the proofs and verified the calculation. All the authors read and approved the final manuscript.

\section{Acknowledgements}

The authors are very grateful to the anonymous referees for their valuable suggestions. This work was supported by the NSFC (No. 11061030), NSFC (No. 11126296), SRFDP (No. 20126203110004) and Gansu Provincial National Science Foundation of China (No. 1208RJZA258)

Received: 15 October 2012 Accepted: 29 April 2013 Published: 15 May 2013

\section{References}

1. Brown, KJ, Budin, $\mathrm{H}$ : On the existence of positive solutions for a class of semilinear elliptic boundary value problems. SIAM J. Math. Anal. 10(5), 875-883 (1979)

2. Peitgen, $\mathrm{HO}$, Saupe, D, Schmitt, K: Nonlinear elliptic boundary value problems versus their finite difference approximations: numerically irrelevant solutions. J. Reine Angew. Math. 322, 74-117 (1981)

3. Peitgen, $\mathrm{HO}$, Schmitt, K: Global topological perturbations of nonlinear elliptic eigenvalue problems. Math. Methods Appl. Sci. 5(3), 376-388 (1983)

4. Hess, P: On multiple positive solutions of nonlinear elliptic eigenvalue problems. Commun. Partial Differ. Equ. 6(8), 951-961 (1981)

5. Ambrosetti, A, Hess, P: Positive solutions of asymptotically linear elliptic eigenvalue problems. J. Math. Anal. Appl. 73(2), 411-422 (1980)

6. Cosner, C, Schmitt, K: A priori bounds for positive solutions of a semilinear elliptic equation. Proc. Am. Math. Soc. 95(1), 47-50 (1985)

7. Dancer, EN, Schmitt, K: On positive solutions of semilinear elliptic equations. Proc. Am. Math. Soc. 101(3), 445-452 (1987)

8. Espinoza, PC: Positive ordered solutions of a discrete analogue of a nonlinear elliptic eigenvalue problem. SIAM J. Numer. Anal. 31(3), 760-767 (1994)

9. Anuradha, V, Shivaji, R: Existence of infinitely many nontrivial bifurcation points. Results Math. 22(3-4), $641-650$ (1992)

10. Anuradha, V, Hai, DD, Shivaji, R: Existence results for superlinear semipositone BVP's. Proc. Am. Math. Soc. 124(3), 747-763 (1996)

11. de Figueiredo, DG: On the existence of multiple ordered solutions of nonlinear eigenvalue problems. Nonlinear Anal. 11(4), 481-492 (1987)

12. Lin, S-S, Pai, FM: Existence and multiplicity of positive radial solutions for semilinear elliptic equations in annular domains. SIAM J. Math. Anal. 22(6), 1500-1515 (1991)

13. Clément, P, Sweers, G: Existence and multiplicity results for a semilinear elliptic eigenvalue problem. Ann. Sc. Norm. Super. Pisa, Cl. Sci. 14(1), 97-121 (1987)

14. Loc, NH, Schmitt, K: On positive solutions of quasilinear elliptic equations. Differ. Integral Equ. 22(9-10), 829-842 (2009)

15. Reichel, W, Walter, W: Radial solutions of equations and inequalities involving the $p$-Laplacian. J. Inequal. Appl. 1(1) 47-71 (1997)

16. Zhang, M: Nonuniform nonresonance of semilinear differential equations. J. Differ. Equ. 166(1), 33-50 (2000)

doi:10.1186/1687-2770-2013-125

Cite this article as: Ma et al.: On positive solutions to equations involving the one-dimensional $p$-Laplacian. Boundary Value Problems 2013 2013:125. 